EPJ Web of Conferences 41, 04006 (2013)

DOI: $10.1051 /$ epjconf/20134104006

(C) Owned by the authors, published by EDP Sciences, 2013

\title{
Ultrafast electronic dynamics in laser-excited crystalline bismuth
}

\author{
A. Melnikov ${ }^{1}$, O. Misochko ${ }^{2}$ S. Chekalin ${ }^{1}$ \\ ${ }^{1}$ Institute of Spectroscopy Russian Academy of Sciences, Troitsk, Moscow Region, 142190, Russia \\ ${ }^{2}$ Institute of Solid State Physics Russian Academy of Sciences, Chernogolovka, Moscow Region, \\ 142432, Russia
}

\begin{abstract}
Femtosecond spectroscopy was applied to capture complex dynamics of nonequilibrium electrons in bismuth. Data analysis reveals significant wavevector dependence of electron-hole and electron-phonon coupling strength along the $\Gamma-T$ direction of the Brillouin zone.
\end{abstract}

\section{Introduction}

Bismuth is a semimetal with unique electronic properties, which are to the large extent governed by symmetry considerations inherent for Peierls-distorted crystals. However, unlike many other elemental crystals, ultrafast electronic dynamics in bismuth attracted less attention than it actually deserves. Femtosecond optical pump-probe experiments performed with bismuth so far generally focused upon detection of coherent phonons, treating electronic response only as a background. If nevertheless decay rates of nonequilibrium electrons were addressed, both the reported values and the interpretation vary from paper to paper. However, detailed knowledge of ultrafast electronic dynamics of bismuth is necessary for a number of theoretical and practical questions. First of all it is needed for a deeper understanding of transient structural phenomena: coherent phonons [1] and ultrafast disordering [2]. Information on the electron-phonon interactions in the elemental crystal is useful for studies of composite systems, e.g. bismuth-antimony compounds [3] and bismuth based nanostructures (such as ultrathin films, nanowires and nanotubes [4]).

Here we report characteristic time values for the relaxation of nonequilibrium charge carriers in bismuth. Observed electronic decay was found to be correlated with both coherent and non-coherent lattice dynamics, spanning a wide range from pico- to nanoseconds. Specific features of the photoinduced response in the visible and NIR domain were interpreted referring to the band structure of bismuth. Thereby it was possible to evaluate electron-hole recombination and electron-phonon coupling rates for certain regions of the Brillouin zone.

\section{Experimental details}

The reliable interpretation of ultrafast dynamics in bismuth was enabled by the availability of timeresolved data in a broad range of wavelengths. We used 70 fs excitation pulses delivered by a parametric amplifier with central wavelength tunable from 400 to $2500 \mathrm{~nm}$. A typical pump-probe layout was implemented to measure photoinduced reflectivity response of a monocrystalline bismuth

This is an Open Access article distributed under the terms of the Creative Commons Attribution License 2.0, which permits unrestricted use, distribution, and reproduction in any medium, provided the original work is properly cited. 
sample. To record $\Delta R / R(t)$ values simultaneously at multiple spectral points femtosecond supercontinuum was generated in a water cell. All measurements were done at room temperature.

\section{Results and discussion}

Figure 1 illustrates main features of the photoinduced reflectivity response of bismuth measured almost in the whole visible domain. Near zero delay time reflectivity sharply increases indicating the arrival of a pump pulse, while subsequent relaxation represents a combination of monotonic signal with damped oscillations superimposed. Oscillations are caused by coherent $A_{l g}$ phonons, while monotonic component stands for the decay of photoexcited charge carriers and noncoherent (thermal) lattice processes.
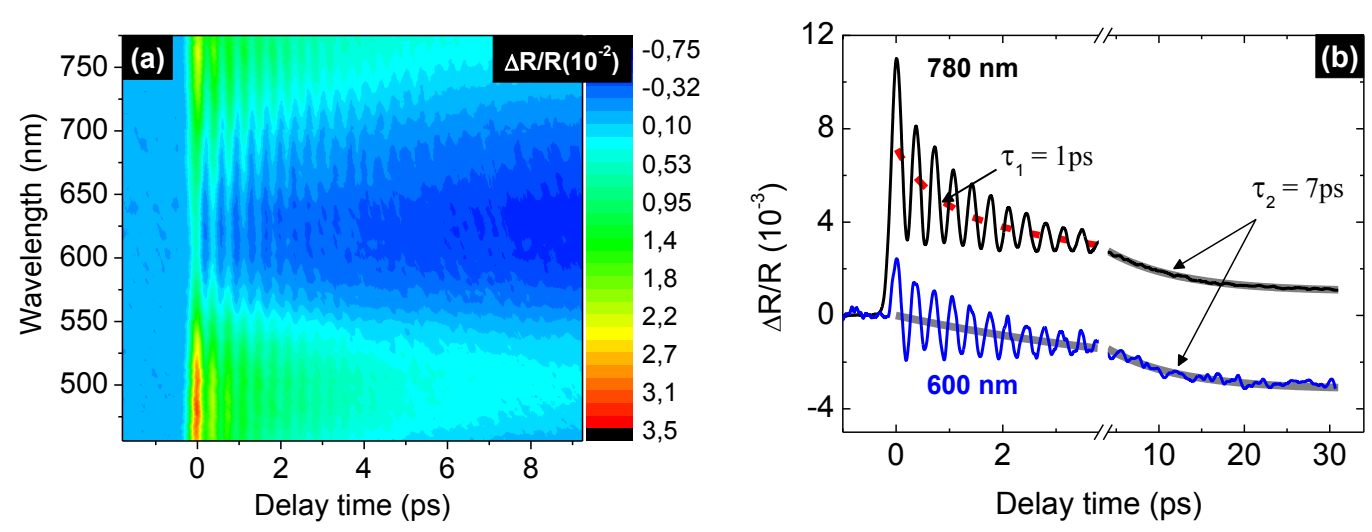

Fig. 1. (a) - Two dimensional array of $\Delta R / R(\lambda, t)$ measured using excitation laser pulses at $400 \mathrm{~nm}$ with the fluence of $1.8 \mathrm{~mJ} / \mathrm{cm}^{2}$. (b) - Decay traces at 600 and $780 \mathrm{~nm}$ measured using single-channel detection and $400 \mathrm{~nm}$ excitation with $\sim 1.3 \mathrm{~mJ} / \mathrm{cm}^{2}$ fluence. Solid lines correspond to slower component of the response while the dashed - to the faster.

We have found that decay traces contain tree distinct exponential components (their amplitudes are referred to as $\sigma_{1}, \sigma_{2}$ and $\sigma_{3}$ ) with characteristic times of $\tau_{1}=1.0 \pm 0.3 \mathrm{ps}, \tau_{2}=7.0 \pm 0.5 \mathrm{ps}$ and $\tau_{3} \sim$ 1 ns. The multi-exponential relaxation is caused by interactions in the electronic subsystem of bismuth and by the flow of energy to the lattice. As follows from the experiments with tunable pump wavelength (Fig. 2(a)), diffusion of charge carriers provides minor contribution to the decay of photoinduced reflectivity in bismuth. This conclusion relies on the comparison of decay traces recorded with $\lambda_{\text {pump }}$ in the range from 400 to $2500 \mathrm{~nm}$. In this range penetration depth increases from $30 \mathrm{~nm}$ to $110 \mathrm{~nm}$ and so does the excitation volume. Keeping the probe volume constant it was possible to evaluate the contribution of transport processes. Additional measurements with almost one order of magnitude fluence variation exclude processes, the rate of which depends on the excited electronic density (e.g. Auger recombination) at least for the picosecond range (for $\sigma_{l}$ and $\sigma_{2}$ ).

The most important result of measurements with variable $\lambda_{\text {pump }}$ is the correlated behavior of $\sigma_{l}$ and oscillatory components: the amplitude of both demonstrates a significant decrease when $\lambda_{\text {pump }}>$ $1800 \mathrm{~nm}(\sim 0.7 \mathrm{eV}$, see the inset in Fig. 2(a)). This is an evidence of strong coupling between electrons having 1 ps lifetime and $A_{l g}$ optical phonons. The comparison of the corresponding threshold photon energy value of $0.7 \mathrm{eV}$ with energies of direct optical transitions in bismuth allowed us to locate $\sigma_{l}$ electrons in a region near $T_{6}{ }^{+}$point of the Brillouin zone. These charge carriers probably cause the generation of coherent $A_{\text {lg }}$ phonons and experience phonon-assisted recombination with holes near $T_{45}{ }^{-}$point.

An insight on the nature of $\sigma_{2}$ and $\sigma_{3}$ components is obtained from their $\lambda_{\text {pump }}$ invariance (Fig. 2(a)), which implies that corresponding charge carriers occupy lower lying states $\left(\varepsilon-\varepsilon_{F}<0.7 \mathrm{eV}\right)$. Additional fact that allows one to make a more concrete conclusion is the similar form of $\sigma_{3}(\lambda)$ and 
$A(\lambda)$ spectra (the latter is the amplitude of coherent oscillations, not shown): both have a minimum near $\lambda_{\text {probe }}=600 \mathrm{~nm}$ and maxima closer to the edges of the $450-780 \mathrm{~nm}$ region. Since coherent $A_{l g}$ phonons in bismuth modulate the indirect overlap of valence and conduction bands, this similarity is natural if we relate $\sigma_{3}$ component to nonequilibrium electrons and holes that reside near the Fermi level in $L$ and $T$ points and experience indirect nanosecond recombination. The $\sigma_{2}$ component then represents their initial metal-like "cooling" in $\sim 7$ ps.
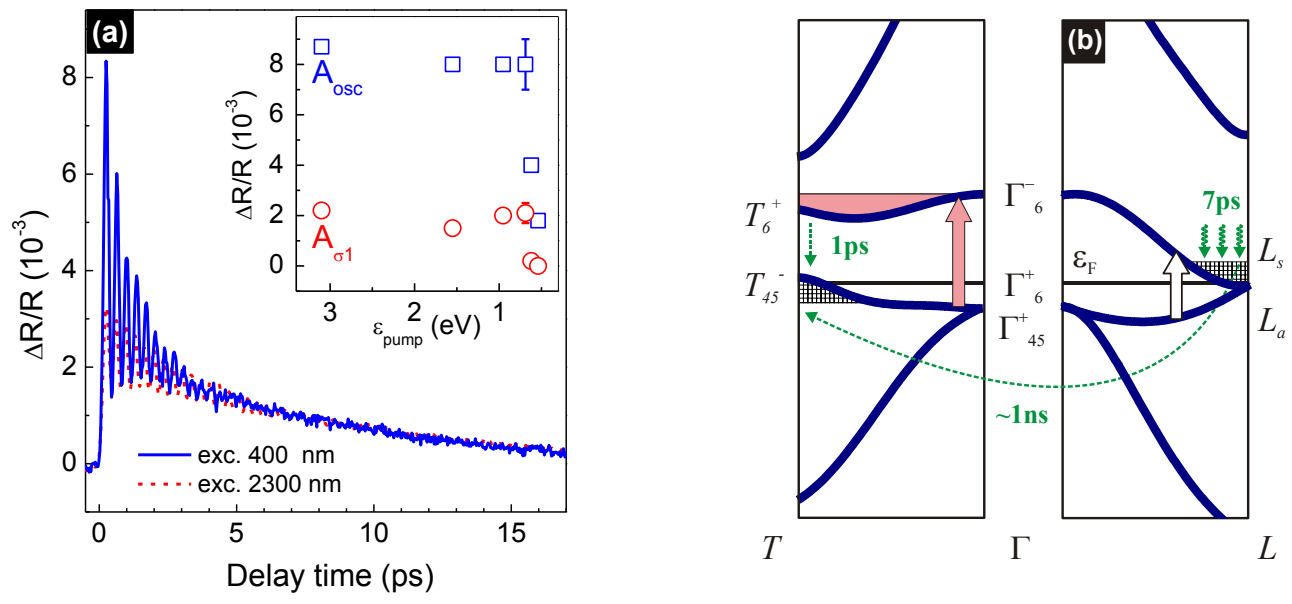

Fig. 2. (a) - Decay traces measured with $400 \mathrm{~nm}$ and $2300 \mathrm{~nm}$ (dashed line) excitation pulses. The inset illustrates pump photon energy dependence of the initial amplitude of oscillations $\left(A_{\text {osc }}\right)$ and the amplitude of the 1 ps component $\left(A_{\sigma l}\right)$. (b) - Part of the band structure of bismuth adapted from [5]. Filled arrow shows resonant excitation of electrons leading to the appearance of fast $1 \mathrm{ps}$ component and generation of coherent $A_{l g}$ phonons. Empty arrow stands for "below-threshold" transitions which cause accumulation of charge carriers near the Fermi level. Dashed regions corresponds to excited electrons ( $L$ point) and holes ( $T$ point) having nanosecond lifetime.

The observed specific features of ultrafast dynamics in bismuth reflect the intermediate position of this material between metals and semiconductors and peculiarities inherent for Peierls-distorted materials. It is clear that frequently used phenomenological [6] and thermodynamic [7] concepts as well as the two-temperature model are too simple to describe coupled electronic and lattice dynamics in bismuth properly. In the present study an alternative interpretation was suggested, according to which several groups of nonequilibrium charge carriers are formed in bismuth after fs-laser excitation. They occupy well defined regions of the Brillouin zone with high density of states, while the subsequent relaxation is defined by the character of electron-hole and electron-phonon interaction.

\section{References}

1. M. Först and T. Dekorsy in, Coherent Vibrational Dynamics, eds. S. D. Silvestri, G. Cerullo, and G. Lanzani (CRC, Boca Raton, 2007), p. 129.

2. G. Sciaini, M. Harb, S.G. Kruglik et al., Nature 458, 56 (2009).

3. D. Hsieh, J.W. McIver, D.H. Torchinsky et al., Phys. Rev. Lett. 106, 057401 (2011).

4. Yu.M. Koroteev, G. Bihlmayer, J. E. Gayone et al., Phys. Rev. Lett. 93, 046403 (2004).

5. S. Golin, Phys. Rev. 166, 643 (1968).

6. H.J. Zeiger, J. Vidal, T.K. Cheng et al., Phys. Rev. B 45, 768 (1992).

7. Y. Giret, A. Gelle, B. Arnaud, Phys. Rev. Lett. 106, 155503 (2011). 